\title{
Thermal Convection Inside Three-Dimensional Differentially Heated Cavity Under Laminar and Transitional Flow Conditions
}

\author{
A. Naveen, R. Harish
}

\begin{abstract}
The study presents the heat transfer phenomena of steady buoyancy driven flows inside a three-dimensional square enclosure. The thermal boundary condition of this enclosure are the vertical side walls are maintained at constant temperature difference and all the other walls are adiabatic. Reynolds averaged Navier stokes (RANS) equations are used to model the flow phenomena inside the enclosure, these equations are discretized using finite difference method (FDM) based Fortran code which was developed in house. The study is done for varying Grashof numbers $10^{5} \leq \mathrm{Gr} \leq 10^{7}$ and a constant Prandtl number 6.2. The results indicated that as the Grashof number increases the temperature along the enclosure decreases by $24.2 \%$ and the rate of transfer of heat inside the enclosure increased by $26 \%$.

Keywords: Natural convection, Cubical enclosure, Finite difference method, Transient flow, Grashof number
\end{abstract}

\section{INTRODUCTION}

Natural convection heat transfer has many applications in engineering fields such as cooling of electronics, solar water heaters, thermal plants etc. Hence there has been an extensive numerical and experimental research in this field of natural convection or buoyancy driven flows in enclosed surfaces. Khalifa et al [1] performed studies on transfer of heat due to natural convective in two-dimensional and three-dimensional enclosures with applications on buildings. Horikiri et.al [2] presented a study on natural convection heat transfer inside a room with a heat source for two-dimensional non ventilated and three-dimensional ventilated cases. The results showed that the position of the source of heat and window had lot of influence in temperature distribution and also the thickness of wall, its conductivity had an influence of thermal comfort of the occupant in the room. November and Nansteel [3] studied numerically and analytically on natural convection heat transfer of water in rectangular enclosure with its bottom wall heated and one of its vertical wall cooled. Corcione [4] performed numerical study on natural convection of 2D rectangular cavities with air, in which the lower wall is heated, upper wall is kept cooled and the side walls are maintained at

Revised Manuscript Received on April 25, 2020.

*Correspondence Author

A. Naveen, Postgraduate Student, M.Tech(CAD/CAM), School of Mechanical Engineering, Vellore Institute of Technology, Chennai, Tamilnadu-600127, India.

R. Harish*, Assistant Professor, Thermal and Automotive Research Group, School of Mechanical Engineering, Vellore Institute of Technology, Chennai, Tamilnadu-600127, India. Email: harish.r@vit.ac.in

(C) The Authors. Published by Blue Eyes Intelligence Engineering and Sciences Publication (BEIESP). This is an open access article under the CC BY-NC-ND license (http://creativecommons.org/licenses/by-nc-nd/4.0/) different thermal boundary conditions. Corvaro et al. [5] performed an experimental study on square cavity filled with air at atmospheric pressure for natural convection heat transfer. In the study on the vertical walls he varied the boundary conditions by using hot and cold strips, three test cases were performed by varying the position of cold strip from bottom, top and middle of the side wall and placing the hot strip in the middle of the opposite side wall. The results indicated that for top configuration the average Nusselt number is highest. Fusegi et al. [6] performed a three-dimensional numerical study of buoyancy driven flows in a cubical cavity. The vertical side walls were at constant temperature difference and the horizontal walls were maintained at different thermal boundary conditions for different cases, the first case was adiabatic and the second case was perfectly conducting. The results indicated that the transfer of heat intensified for conducting horizontal walls. Sezai and Mohamad [7] performed two-dimensional and three-dimensional simulations for fluid flow in open cubical cavity in which one of the vertical walls is heated, the opposite wall is open and all other walls are insulated at Rayleigh numbers of $10^{3}$ to $10^{6}$, constant Prandtl number 0.71 (air) and varying lateral aspect ratio. The air surrounding enters inside the cavity from bottom and leaves from top of the vertical opened wall due to natural convection. The results indicated that heat transfer rate of two-dimensional model is similar to three-dimensional model for $\mathrm{Ra}<10^{5}$. But for greater vales of $\mathrm{Ra}$ the two-dimensional model did not with the three-dimensional model results hence for $\mathrm{Ra}>10^{5}$ three-dimensional model is necessary to predict heat transfer rate of open channels. Paroncini et al. [8] presented a numerical and experimental study of air flow due to natural convection in square cavities with its vertical side walls kept at different temperatures at different locations. They conducted experimental test cases with different heat and cold sources with different lengths on the side wall to study its effect of heat transfer in natural convection. The results indicated an increase of the Nusselt numbers to the Rayleigh numbers. And also, as the number of heat sources on the side wall increased irrespective of the source length the heat transfer increases. Oliveski et al. [9] numerically investigated the entropy generation in rectangular enclosures subjected to buoyancy driven flows. The boundary conditions in this study was one of the vertical wall is hot, the opposite wall is cold and all the other walls are insulated. The results indicate that in steady state as the aspect ratio increases the total entropy generation increases linearly, also exponentially with Rayleigh number. 
Frederick and Berbakow [10] performed numerical analysis of natural convection in a cubical enclosure with one of the wall as a heat source and the adjacent wall is cooled for a Rayleigh numbers range of $10^{4}-10^{6}$. For $\mathrm{Ra}<10^{5}$, the transfer of heat mostly hinged on heated wall than the dependence on Rayleigh number. But for higher $\mathrm{Ra}$ numbers this was opposite. The present study investigates the transfer of heat for steady natural convection flow in cubical enclosure. The side walls are maintained at a constant temperature difference and the other wall are kept adiabatic. This study is modeled based on the RANS equations of mass, momentum and energy conservation. This study is carried out for a range of Grashoff number $10^{4}<\mathrm{Gr}<10^{7}$ with prandtl number being constant at 6.2. Temperature and streamline contours are analyzed for various Grashof numbers. The numerical method used is a Finite difference (FDM) solved using a in-house Fortran code.

\section{GOVERNING EQUATIONS AND NUMERICAL METHOD}

The schematic diagram of the current study is depicted in Figure 1 . The model is a three-dimensional cubic cavity of length $1 \mathrm{~m}$ whose two opposite sides are kept at a differential temperature and the other sides are adiabatic. The numerical simulations are performed by using a Finite difference based [11-14] (FDM) Fortran code, where the problem is modeled as natural convection flow. The flow is modeled using Reynolds Averaged Navier-Stokes (RANS) equations [15,16] of steady three-dimensional natural convection flow expressing mass, momentum, and energy conservation equations.

$\frac{\partial u}{\partial x}+\frac{\partial v}{\partial y}+\frac{\partial w}{\partial z}=0$

$$
\begin{gathered}
\rho\left(u \frac{\partial u}{\partial x}+v \frac{\partial u}{\partial y}+w \frac{\partial u}{\partial z}\right)=-\frac{\partial p}{\partial x}+ \\
\mu\left(\frac{\partial^{2} u}{\partial x^{2}}+\frac{\partial^{2} u}{\partial y^{2}}+\frac{\partial^{2} u}{\partial z^{2}}\right)
\end{gathered}
$$

$\rho\left(u \frac{\partial v}{\partial x}+v \frac{\partial v}{\partial y}+w \frac{\partial v}{\partial z}\right)=-\frac{\partial p}{\partial y}+$

$\mu\left(\frac{\partial^{2} v}{\partial x^{2}}+\frac{\partial^{2} v}{\partial y^{2}}+\frac{\partial^{2} v}{\partial z^{2}}\right)$

$\rho\left(u \frac{\partial w}{\partial x}+v \frac{\partial w}{\partial y}+w \frac{\partial w}{\partial z}\right)=-\frac{\partial p}{\partial z}+$

$\mu\left(\frac{\partial^{2} w}{\partial x^{2}}+\frac{\partial^{2} w}{\partial y^{2}}+\frac{\partial^{2} w}{\partial z^{2}}\right)$

$u \frac{\partial T}{\partial x}+v \frac{\partial T}{\partial y}+w \frac{\partial T}{\partial z}=\alpha\left(\frac{\partial^{2} T}{\partial x^{2}}+\frac{\partial^{2} T}{\partial y^{2}}+\frac{\partial^{2} T}{\partial z^{2}}\right)$

Where ' $\rho$ ' represents the density of fluid, ' $u, v, w$ ' are the velocity fields, ' $\mathrm{T}$ ' represents temperature field, ' $\mu$ ' represents viscosity and ' $\alpha$ ' represents thermal diffusivity. The strength of buoyancy flow is described by using a non-dimensional number called Grashof number (Gr). The Grashof number signifies the effect of force due to buoyancy over the force due to viscosity.

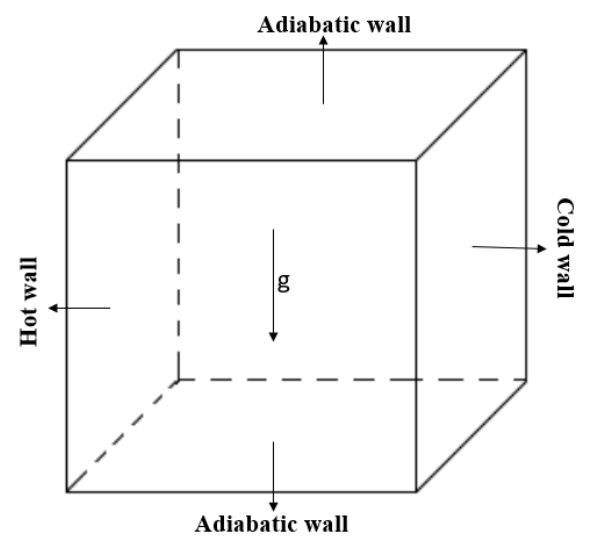

Figure 1: Three-dimensional differentially heated cubical $G r=\frac{g \beta \Delta T L^{3}}{v^{2}}$

where ' $g$ ', ' $\beta$ ', ' $\Delta \mathrm{T}$ ', ' $L$ ' and ' $v$ ' represent gravity, thermal expansion co-efficient of fluid, temperature difference, length of the enclosure and kinematic viscosity of the fluid.

\section{RESULTS AND DISCUSSION}

Figure 2 presents the streamlines of the flow at time intervals 50,100 for $\mathrm{Gr} 10^{7}$. It can be observed that the fluid rises from bottom along the left wall then circulate along the top and right wall to form a vortex motion due to the temperature difference (buoyancy flow). And also, as the time increases streamlines tend to move further apart this is due to the reduction in temperature difference at middle of the enclosure.
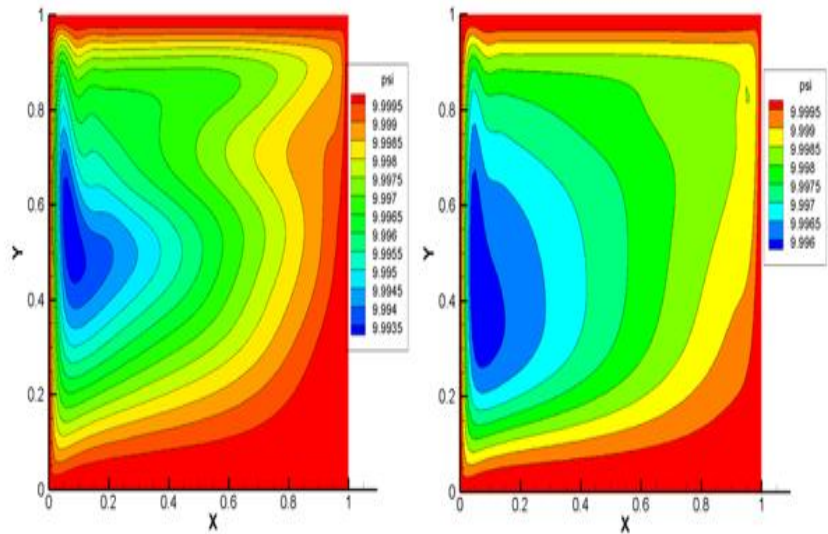

Figure 2: Streamlines for $\mathrm{Gr}=10^{7}$ at time intervals 50 and 100

Figure 3 shows the temperature contours of a flow with $\mathrm{Gr}=10^{7}$ at different time intervals. It can be observed that the temperature in the cavity increases from top left to the middle of the enclosure due to the circulation of flow over a period of time. Figure 4 shows the 3D iso surfaces of temperature for $\mathrm{Gr}=10^{6}$ at different time intervals. We observe that as the time passes, temperature distribution intensifies across the enclosure due to the circulating flow.

Published By:

Blue Eyes Intelligence Engineering \& Sciences Publication

(C) Copyright: All rights reserved.

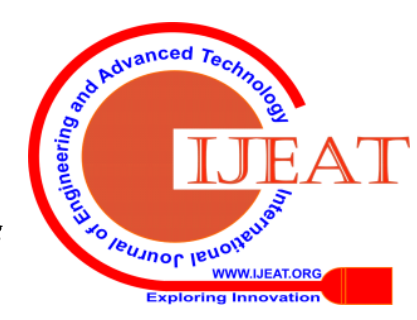



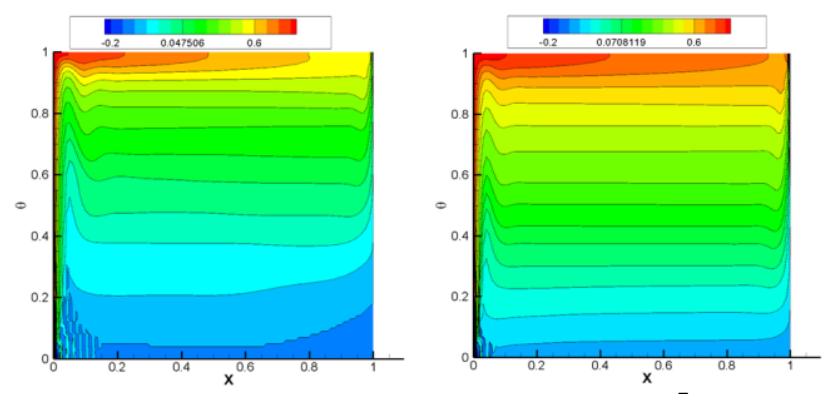

Figure 3: Temperature contour for $\mathrm{Gr}=10^{7}$ at time intervals 50 and 100
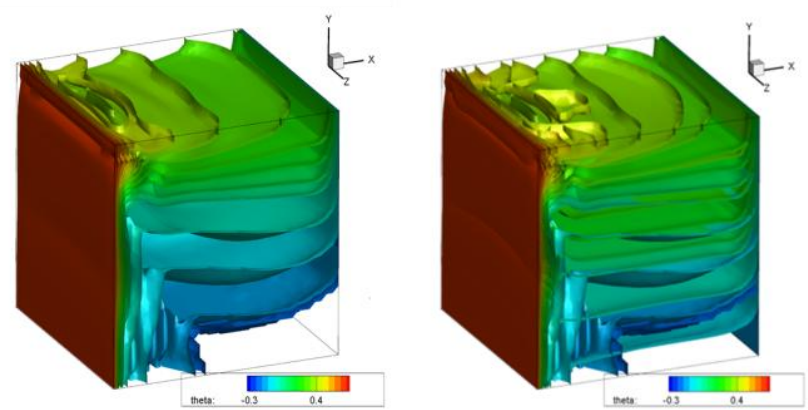

Figure 4: Iso surfaces of temperature for $\mathrm{Gr}=10^{6}$ at time intervals 25 and 50

Figure 5 shows the variation Temperature at mid-plane for $\mathrm{Gr}=10^{7}$ at different time intervals of 25, 50, 75 and 100. It is observed that as the time interval progresses from 25 to 100 there is gradual increase in temperature distribution at the mid plane of the enclosure, which is equal 15\%. Figure 6 presents variation of $\mathrm{V}$ - velocity of flow at the mid plane for $\mathrm{Gr}=10^{7}$ at different time intervals of 25, 50, 75 and 100. We can observe that the fluid flow velocity is high at the left side of enclosure due to high temperature and the velocity tends to drop as we progress from left to right side of enclosure due to the reduction in temperature.

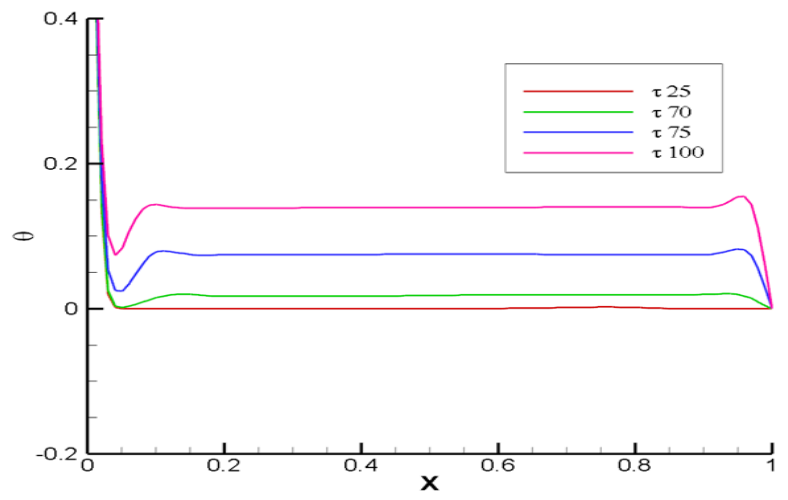

Figure 5: Temperature variation at mid plane for $\mathrm{Gr}=10^{7}$ and $P r=6.2$ at time intervals 25, 50, 75 and 100

Figure 7 presents Temperature variation at mid plane for different Grashof numbers, Gr=10 $10^{6}, 10^{7}$.we can observe that as the $\mathrm{Gr}$ number increases there is a decrease in temperature and this is $24.2 \%$ decrease. As Gr number increases, there is domination of buoyancy force over the viscous forces which tends the fluid to cover more area there by increasing the heat transfer rate and decreasing the temperature in the enclosure.

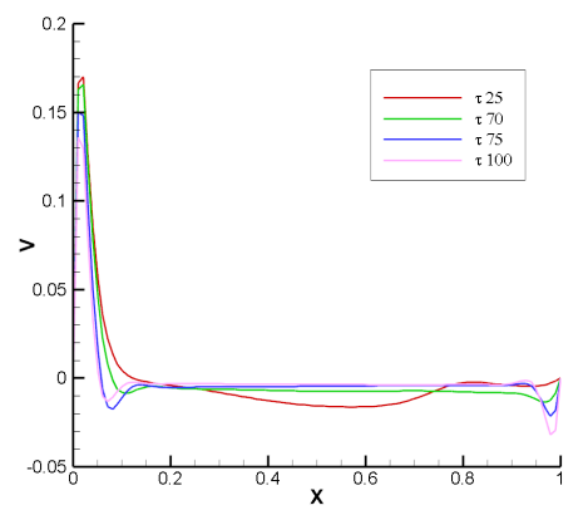

Figure 6: V-velocity variation at mid plane for $\mathrm{Gr}=10^{7}$ at time intervals 25, 50, 75 and 100

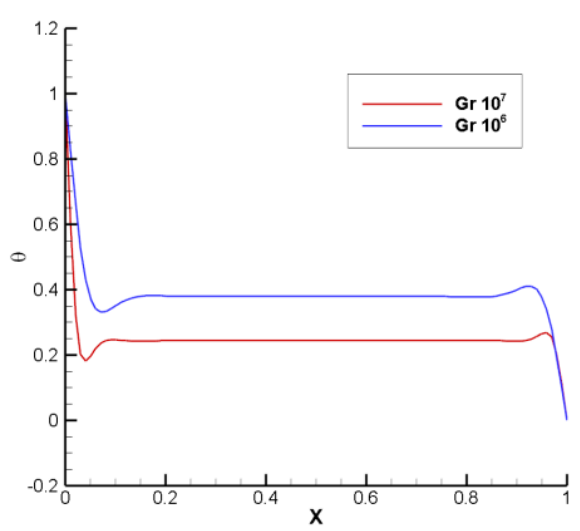

Figure 7: Temperature variation at mid plane for $\mathbf{G r}=10^{6}$ , $\mathbf{1 0}^{7}$

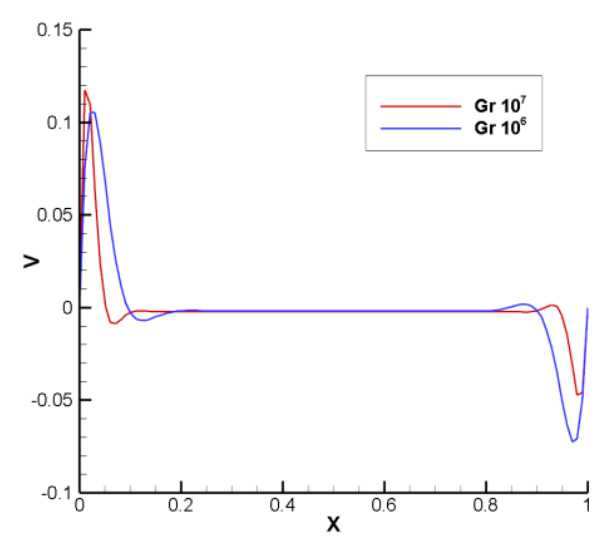

Figure 8: V- velocity variation at mid plane for $\mathrm{Gr}=10^{6}$ , $\mathbf{1 0}^{7}$

Figure 8 presents the variation $\mathrm{V}$ - velocity of flow along the mid plane for $\mathrm{Gr}=10^{5}, 10^{7}$. Here we can clearly observe that as the Grashof number increases the velocity changes instantly due to increased buoyancy force on the flow, there by implying the sharp variation of velocity for higher $\mathrm{Gr}$ numbers.

Published By:

Blue Eyes Intelligence Engineering \& Sciences Publication

(C) Copyright: All rights reserved.

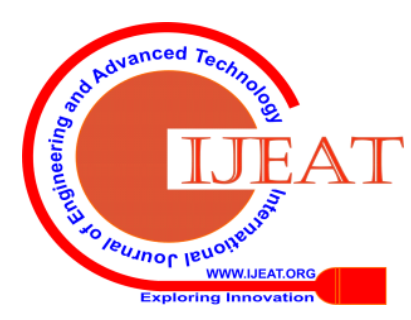




\section{CONCLUSION}

Steady natural convection flow characteristics inside a cubical enclosure with one side hot the opposite side cold and all the other sides are maintained adiabatic, is numerically investigated by varying Grashof number Gr. The flow is modeled by using Reynolds averaged Navier Stokes equations (RANS) of mass, momentum and energy equations and these equations are solved using finite difference method-based FORTRAN code. The streamlines and temperature profiles are plotted for different Grashof numbers at different time intervals. The streamlines indicate the formation of vortex flow along the hot surface and then spread as the time progresses. It is also seen that due to this vortex flow the temperature in the enclosure gradually increases from left to right side. And as the Grashof number increased the temperature decreased by $24.2 \%$, and the heat transfer rate inside the enclosure increased by $26 \%$.

\section{REFERENCES}

1. A. Khalifa, Natural convection heat transfer coefficient - a review II Surface in two- and three-dimensional enclosures, Energy Conversion and Management. 2001, pp:505-517.

2. K. Horikiri, Y. Yao, J.Yao, Modelling conjugate flow and heat transfer in a ventilated room for indoor thermal comfort assessment, Building and Environment, 2014,pp:135-147.

3. M. November, M. Nansteel, Natural convection in rectangular enclosures heated from below and cooled along one side, Int. J. Heat Mass Transf. 1987, pp: 2433-2440.

4. M. Corcione, Effects of the thermal boundary conditions at the sidewalls upon natural convection in rectangular enclosures heated from below and cooled from above, Int. J. Therm. Sci. 2003, pp:199-208.

5. F. Corvaro, M. Paroncini, M. Sotte, Experimental PIV and interferometric analysis of natural convection in a square enclosure with partially active hot and cold walls, Int. J. Therm. Sci. 2011, pp: 1629-1638.

6. T. Fusegi, J. Hyun, K. Kuwahara, A numerical study of 3D natural convection in a cube: effects of the horizontal thermal boundary conditions, Fluid Dyn. Res. 1991, pp:221-230.

7. I. Sezai, A. Mohamad, Three-dimensional simulation of natural convection in cavities with side opening, Int. J. Numer. Methods Heat Fluid Flow 8 (7) 1998

8. M. Paroncini, F. Corvaro, A. Montucchiari, G. Nardini, A numerical and experimental analysis on natural convective heat transfer in a square enclosure with partially active side walls, Exp. Therm. Fluid Sci. 2012, pp:118-125.

9. R. Oliveski, M. Macagnan, H. Macagnan, J. Copetti, Entropy generation and natural convection in rectangular cavities, Appl. Therm. Eng. 2009, pp:1417-1425.

10. R. Frederick, O. Berbakow, Natural convection in cubical enclosures with thermal sources on adjacent vertical walls, Numer. Heat Transf. Part A 41 (3), 2002, pp:331-340.

11. R.Harish, Buoyancy driven turbulent plume induced by protruding heat source in vented enclosure, International Journal of Mechanical Sciences,2018,pp:209-222.

12. R.Harish, Effect of heat source aspect ratio on turbulent thermal stratification in a naturally ventilated enclosure, Building and Environment, 2018,pp:473-486 .

13. R.Harish,K.Venkatasubbaiah, Non-Boussinesq approach for turbulent buoyant flows in enclosure with horizontal vent and forced inlet port, Applied Mathematical Modelling, 2016,pp:927-941.

14. R.Harish, K.Venkatasubbaiah, Numerical investigation of instability patterns and nonlinear buoyant exchange flow between enclosures by variable density approach, Computers \& Fluids, 2014,pp:276-287.

15. R.Harish, K.Venkatasubbaiah, Mathematical modeling and computation of fire induced turbulent flow in partial enclosures,AppliedMathematicalModelling,2013,pp:9732-9746.

16. R.Harish,K.Venkatasubbaiah, Numerical simulation of turbulent plume spread in ceiling vented enclosure, European Journal of Mechanics-B/Fluids,2013,pp:142-158.

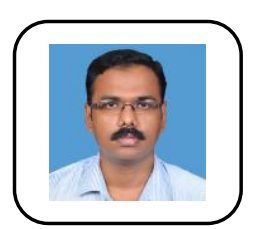

\section{AUTHORS PROFILE}

Dr. R. Harish is working as an Assistant Professor in the school of Mechanical Engineering at VIT Chennai campus. His research interests are in the field of computational fluid dynamics, Heat Transfer and Turbulent flows

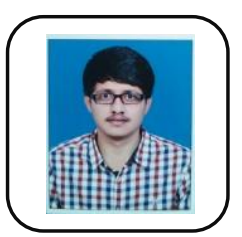

Naveen Kumar A is pursuing M-tech CAD/CAM in the school of Engineering at VIT Chennai campus with an Undergraduate degree in Mechanical engineering from NMIT Bangalore.

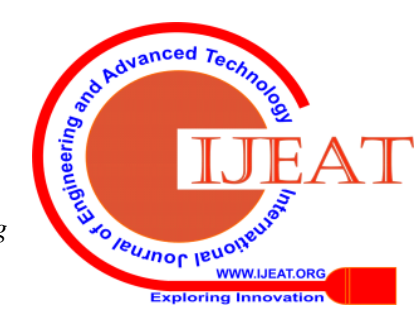

